\title{
Apple Peel Atresia with Isolated Fetal Ascites and Digit Anomalies
}

\author{
Can Ozlu ${ }^{1}$ (1) $\cdot$ Mehmet Ali Ozen $^{2} \cdot$ Tugba Gursoy $^{3} \cdot$ Pelin Oguzkurt $^{2}$
}

Received: 1 October 2018 / Accepted: 21 February 2019 / Published online: 6 March 2019

(C) Dr. K C Chaudhuri Foundation 2019

To the Editor: We present a case of isolated massive fetal ascites associated with apple peel intestinal atresia and unique digit anomalies in which prenatal ultrasonography findings such as bowel dilatation, intraabdominal calcifications and increased bowel echogenicity were absent. He was born to a 32-y-old gravida 1 para 1 mother (nonconsanguineous parents) via $\mathrm{C}$-section at the gestational age of $32 \mathrm{wk}$ due to preterm labor related to severe polyhydramnios. Prenatal history revealed isolated fetal ascites in the 16 th wk of gestation. Amniocentesis showed normal karyotyping, cystic fibrosis genetic screening and infection screen were negative. At birth he had severe abdominal distention due to massive ascites causing respiratory distress. The birth weight was $1600 \mathrm{~g}$ (10-50p), height was $40 \mathrm{~cm}$ (10p) and the head circumference was $29.5 \mathrm{~cm}$ (50p). Anomalies were noted in the hands and feet; with brachydactyly, anonychia/onychodystrophy of multiple fingers of both hands and syndactyly of 2 nd and 3rd toes of the right foot. Urgent laparotomy was performed because of pneumoperitoneum on the first day of life, revealing $300 \mathrm{ml}$ of clear serous fluid and isolated jejunoileal atresia type IIIB (apple peel atresia) with $20 \mathrm{~cm}$ proximal and $6 \mathrm{~cm}$ distal small intestinal segments. The proximal perforated atretic bowel end and distal atretic bowel end were resected and primary anastomosis was performed. The multiple digit anomalies

Can Ozlu

cozlu@ku.edu.tr

1 Koc University School of Medicine, Koc University Hospital, 4 Davutpasa Street, Topkapi, 34010 Istanbul, Turkey

2 Department of Pediatric Surgery, Koc University Hospital, Istanbul, Turkey

3 Department of Pediatrics, Koc University Hospital, Istanbul, Turkey were confirmed with skeletal survey. Unfortunately, he developed cholestasis and severe sepsis in the postoperative period and died.

Isolated fetal ascites, found on prenatal ultrasound is generally a sign of underlying congenital malformation, with approximately $25 \%$ involving the gastrointestinal (GI) system [1]. The early development of ascites in the neonate (in the 16th wk) could be indicative of ascites due to an early vascular insult and/or in-utero bowel perforation. Early isolated fetal ascites detected before 24th gestational week is associated with a significantly increased mortality risk and produces worse postnatal outcome [2]. The combined presentation of GI and digit malformations has not been described in the literature previously. This case demonstrates that intestinal atresia should be kept in mind in differential diagnosis of isolated fetal ascites even when signs of intrauterine intestinal obstruction are absent.

Authors' Contributions $\mathrm{CO}$ collected the clinical data and wrote the manuscript. All the authors conceptualized the report, supervised manuscript writing and reviewed the manuscript. All authors approved the final manuscript as submitted and agree to be accountable for all aspects of the work. PO will act as guarantor for this paper.

\section{Compliance with Ethical Standards}

Conflict of Interest None.

Publisher's Note Springer Nature remains neutral with regard to jurisdictional claims in published maps and institutional affiliations.

\section{References}

1. Schmider A, Henrich W, Reles A, Kjos S, Dudenhausen JW. Etiology and prognosis of fetal ascites. Fetal Diagn Ther. 2003;18: 230-6.

2. Catania VD, Muru A, Pellegrino M, et al. Isolated fetal ascites, neonatal outcome in 51 cases observed in a tertiary referral center. Eur J Pediatr Surg. 2017;27:102-8. 